\title{
Review of: "A new long-term sampling approach to viruses on surfaces"
}

\author{
Shubhada Bopegamage
}

Potential competing interests: The author(s) declared that no potential competing interests exist.

The authors have assessed a new paper-based sticker method for collecting samples from fomites to check for viral contamination and also compared the method to the classical swab collection especially for testing viral loads by measuring the plaque forming units and the viral genome copies by realtime PCR. The authors show that the new method is efficient.

The idea is good. It is space and time saving also economic.

The figure and tables are clear.

But I have few comments and questions.

In the methods section - Cleaning and disinfection of surfaces is clear but the section Artificial contamination of stickers, swabs and controls title should be changed. Here the authors are testing the ability of absorption of known virus.

1. Were different dilutions tried out here? If yes this should be mentioned clearly. Volume and virus titer.

2. This should be clear also in the section Cleaning and disinfection of surfaces.

3. If the sticker is present for a longer time and the surface is cleaned at different times, I cannot understand the mechanism how the after-treatment surface is checked, is the sticker removed and stuck in another place?

4. How will you check prior detergent exposed surface? Do you eluate the virus or the viral DNA and then re-stick the tape.

5. A photograph of the actual tape would help the readers. 